\title{
Research on Human Capital Development Strategy of New-Type Professional Farmers under the Background of Hollow Rural Population
}

\author{
Shu-qin TIAN ${ }^{1,2,3}$, Dong-qiang WANG ${ }^{2, *}$ \\ ${ }^{1}$ School of Finance and Economics, \\ Chongqing Vocational College of Transportation, \\ Chongqing \\ ${ }^{2}$ School of public administration, \\ Chongqing University of Arts and Sciences, \\ Chongqing \\ ${ }^{3}$ Postdoctoral Program of Fudan University, \\ *Corresponding author
}

\begin{abstract}
With the rapid development of new urbanization process, new-type professional farmers are the main force to realize agricultural modernization in China. Under the background of rural population hollowing, there is a great shortage of new-type professional farmers in China, which leads to the increasingly prominent contradiction between the development requirements of realizing agricultural modernization.This research mainly adopts the research method of questionnaire survey, supplemented by literature research, field investigation, in-depth interviews, case analysis and other analysis methods to investigate and analyze the current situation and problems of cultivating new-type professional farmers, draw the main dilemma of human capital development of new-type professional farmers, and put forward the main cultivation Strategies of new-type professional farmers.
\end{abstract}

Keywords-population hollowing; new type of professional farmers; human capital; development strategy

\section{INTRODUCTION}

Under the background of rural population hollowing, farmers, as an important stratum to realize agricultural modernization, are the main force to realize the rapid development of the rural economy. Today, with the development of China's economy, the development of rural areas lags far behind that of cities and towns, and has not yet been effectively developed. Therefore, in order to deepen the

Sponsors: This paper was supported by the National Social Science Foundation Project "Research on the Hollow Governance System of Rural Population in Four Centralized and Connected Poverty-stricken Areas" (No. 16CSH041), the Ministry of Education Humanities and Social Science Project "Research on the Overall Governance and Policy Aiming of Training Funds for New Vocational Farmers under the Background of Rural Revitalization" (No. 19XJC880009), the projects of the People's and Social Bureau of Chongqing "Implementing the Strategy of Rural Revitalization and the Research on the Institutional Supply of the Cultivation of New Professional Farmers in Chongqing", "Research on Human Capital Development of New-type Professional Farmers in Chongqing under the Strategic Background of Rural Revitalization” commissioned by Chongqing Social Science Planning Project,"Research on the Evaluation System and Standards for the Revitalization of Rural Talents" commissioned by Yongchuan District People's and Social Bureau and the PhD Project of Social Science Planning of Chongqing (No. 2018BS48). rural reform and build a new agricultural management system, we must transform the traditional class of farmers and realize their professionalization. Farmers' professionalization is the process of professional farmers. To achieve this process, we must train a group of new-type professional farmers who are called new-type professionalized farmers refers to the gradual departure from the natural and semi-natural economic conditions of production and lifestyle, thinking mode and values, to achieve modernization, industrialization, commercialization of production methods and urbanization, the transformation of the times, from a traditional peasant identity to professional identity. Through the investigation and practice of peasant college, some problems and difficulties have been found in the process of cultivating new-type professional peasants[1]. Our government also attaches great importance to the cultivation of new-type professional farmers. Vigorously cultivating new-type professional farmers is also the fundamental way to solve the problem of "who will plant the land, how to plant it well, and how to do a good job in rural economy".

\section{PRoBlems of Human CAPITAl DeVElopment OF NEW-TYPE PROFESSIONAL FARMERS}

\section{A. Low educational level and weak awareness of active participation in training}

Cultivating new-type professional Farmers is the foundation and guarantee for the first realization of agricultural modernization [2]. According to the questionnaire survey of human capital development of new-type professional farmers, 70.99\% of the agricultural practitioners are below middle school culture, and most of them are farmers over 40 years old. The average age of farmers is 34.8 years old. Women are also the main group among the young and middle-aged. About $80.51 \%$ of the farmers say that their new-type professional farmers are reforming at present. The main difficulty of manufacturing and income improvement is low educational years. Lack of professional knowledge and skills is one of the 
important restrictive factors that restrict the expansion of production and the development of human capital of new-type professional farmers. Some family farmers and managers of agricultural enterprises have become new-type professional farmers because of their need and ability to receive new knowledge and training, and they regularly participate in various types of training at all levels, with high professional quality. Most farmers, due to age, cultural level and other reasons, lack of awareness of active participation in training, or lack of time and energy to participate in training because of unstable work, fear that training can not quickly affect employment, work and income, and sometimes there is a certain degree of resistance, the gap between farmers is relatively large, but also heavy. One of the problems to be solved in carrying out the training of farmers'skills is participation consciousness.

\section{B. Insufficient training resources and poor propaganda}

Based on the analysis of relevant data and survey data, it is found that the overall supply of resources for farmers' training in Chongqing is still insufficient. The farmer training project not only involves a large number of peasant groups but also involves the agricultural committee, women's federation, township and other departments. Although the total amount of training investment in society is large, the implementation of each training institution, human, material and financial investment is insufficient. National policies benefit most farmers, but the total number of farmers is relatively large. Policies may also be hampered by some difficulties. Of the 131 farmers in the sample, 47 (35.88\%) farmers have not participated in the training in recent years, while only 45 (34.35\%) farmers have participated in 1-3 times, and most of them have relatively high educational level. Only 11 (8.0\%) have trained more than 6 times $(4.0 \%)$. It can be seen that the participation rate of farmers in Chongqing is not very high. According to the survey and statistical analysis, some farmers in 12 districts and counties of Chongqing preferred to participate in the development of human capital of new-type professional farmers through the business ability of local government organizations and agricultural cooperatives, in order to continuously improve their professional skills (26.2\%) and employment skills (22.7\%).

The survey found that $52.48 \%$ of the respondents believed that the government and village committee had the greatest impact on their own human capital development, indicating that the training organized by the government and village committee was generally acceptable to farmers, but $71.75 \%$ of the respondents had a general and unsatisfactory attitude towards the learning platform in the village, and $39.69 \%$ of the respondents had a correct attitude towards it. The training of former grass-roots government organizations showed unsatisfactory attitudes, and the respondents as a whole did not understand, understand or even be unsatisfactory with the training of the government, which is obviously the manifestation of the absence of the government.

\section{The training method is single and the training content lacks pertinence}

Because of the government's huge investment averages to a large number of farmers, it seems that resources are less.
Because the public welfare training methods of government organizations benefit farmers, but some training institutions are driven by economic interests or the requirements of higher authorities to train, so the quality of training is often unsatisfactory. At present, training is mostly centralized. Farmers often have no time and energy to participate in vocational training activities because of their livelihood. At the same time, a large proportion of farmers go out to work. They are worried that training can not quickly affect employment, work and income. Therefore, a large number of farmers are unwilling to train at home. It also has a negative impact on the training effect. Moreover, nowadays the training methods are mainly in the form of PPT, presenting the phenomenon that PPT talks to the end.

According to the survey, $63.65 \%$ of the farmers who have participated in the training are not satisfied with the training methods. A large proportion of the farmers have received less training and think that the current training methods are too single. And in the early stage of training, the government and training institutions did not carry out special research. They all listened to what the teachers said after the beginning of training The teaching methods were lack of diversity and pertinence. They did not teach knowledge in-depth on-the-spot. Farmers' knowledge and the cultural level was relatively low, and the theoretical content of teaching might not be able to meet. Fully understand, such as: professional knowledge, employment skills, and so on, and these are exactly what farmers need most, which greatly affects the enthusiasm and efficiency of farmers' learning.

\section{THE SOLUTIONS TO IMPROVE HUMAN CAPITAL DEVELOPMENT OF NEW-TYPE PROFESSIONAL FARMERS}

\section{A. Developing Basic Ability Training to Improve Farmers' Cultural Quality}

The training of new-type professional farmers is not accomplished overnight but has a gradual development process[3]. From the basic characteristics of the sample, we can see that the majority of farmers at this stage have a low educational level. Among the new-ype of professional farmers surveyed, junior high school education is the majority, accounting for $42.75 \%$, college education and above only 11 farmers, accounting for $11.45 \%$. With the development of internet technology, a large number of new technologies and knowledge will be imparted. For example, to promote e-commerce in rural areas, at least we need to use computers to access the Internet. For example, many APP promotions and network distance learning, at least we need to use smartphones. In view of the current situation, the local governments should carry out some information technology education in different levels, classifications and time periods to cultivate basic abilities such as computers, smart phones and basic e-commerce through bottom-up investigation and research. In addition, farmers should be given more opportunities to receive basic education. While popularizing nine-year compulsory education, we should properly increase the enrollment rate of peasants or their children; increase the proportion of rural students in key institutions of higher learning through special national or university plans; encourage them to return home and start businesses after graduation; and combine academic 
education with "new vocational farmers training", and adopt the following measures: training new vocational farmers in the form of affiliated or directional training of colleges and universities; vigorously developing vocational education, introducing new technologies and giving field explanations by senior experts; at the same time, the government should increase its support to farmers' vocational colleges and agricultural colleges and universities, establish a broader absorption mechanism for training subjects, and mobilize them. More people's enthusiasm and improve the main status of farmers.

\section{B. Relying on the Internet to Improve Training Archives and Strengthen the Means of Training and Publicity}

Under the background of modernization, agricultural modernization has developed rapidly with the support of national policies[4]. Education and training is the most direct and effective way to improve farmers' production and management level and quality[5]. With the advent of the Internet era in China, in order to realize the revitalization of agriculture, we also need the help of the internet. According to the new-type professional farmers and the total amount of training in various districts and counties of Chongqing, 47 of the 131 farmers $(35.88 \%)$ have not participated in the training in recent years, but have participated in 1-3 times. Only 45 people (34.35\%) were trained, which shows that training propaganda is not enough. In addition, the relevant departments should also do a good job of archives, use the big data of the internet to establish training archives, and timely feedback, timely understanding of the training effect. Through the analysis of farmers preferences, the vast majority of farmers can participate in different types of training according to their own development characteristics and interests.

\section{Enriching the form of teaching and training content of scientific facilities}

According to the analysis of this questionnaire, the main focus of human capital development of new-type professional farmers is professional knowledge and skills. So for farmers, training institutions should focus on the training to improve the ability and quality of life of farmers. However, $77.09 \%$ of the farmers are willing to receive vocational training for less than 15 days, mostly because of livelihood problems. Most of the farmers go out to work or because of agricultural problems, resulting in training can not better attract farmers. Therefore, in order to strengthen the training effect, the teaching form should be flexibly arranged according to the actual situation of Chongqing urban agriculture. In the idle season, farmers have enough time to devote themselves to training; on the contrary, if training is conducted in busy farming, the teaching time is short and the time is dispersed, the training effect will be greatly reduced. Training institutions can adopt the form of classroom teaching to explain the practical problems encountered in the production process or the practical operation technology in the industry, supplemented by flexible and vivid field teaching, explanation of agricultural parks and incubation bases of agricultural projects, which can not only increase the interest of training, but also aim at the industry technology. To develop knowledge reserve, farmers can also choose relevant agricultural projects to incubate, and get the support of the government.
On the one hand, we need to innovate the training mode and enrich the training content[6].On the other hand, the most important problem of farmers' training is the time limit. Therefore, training institutions can record training videos on the platform of APP software and Wechat Public Number, collect farmers' information in real-time and send the practical operation process or theoretical knowledge needed by different levels of farmers to each of them with pertinence and emphasis. In the hands of participating peasants, and let them choose topics of interest or need and teachers team, or even take the form of "flip classroom" to achieve targeted so that farmers can better understand and master the teaching content, enhance their professional post skills and employment skills, and improve social adaptability.

According to the theory of human capital, the role of new occupation and human capital development of peasants in accelerating the process of people-oriented Rural Revitalization Strategy is mainly embodied in the following aspects: first, the accumulation effect of human capital. In the process of urban migrant workers, the new generation of migrant workers not only nurtured the modern life concept, working procedure and thinking mode, but also improved the wage level and family economic income, accumulated certain work experience and acquired a lot of social knowledge by investing in various aspects of education, vocational training and health care. It improves vocational skills and human capital. On this basis, the new type of professional farmers constitutes the main body of the development and sustainable development of the Rural Revitalization strategy, which effectively promotes the process of Rural Revitalization strategy. The second is the spillover effect of human capital. On the one hand, the development of human capital makes the citizenship and professional ethics of the new type of professional peasants continuously improve, and the degree of modernization and civilization is higher, which also has a positive impact on other rural labor force and urban residents, and effectively improves the level of civilization in the countryside. On the other hand, in the process of transferring from rural to urban areas, the development of human capital has expanded the social network of new-type professional peasants, improved their social communication and public relations capabilities, and provided them with more opportunities to obtain the latest information and employment opportunities, which has enhanced their ability to integrate and utilize social resources. Social capital has been effectively accumulated. Thirdly, the entrepreneurial effect of human capital. As mentioned above, the dual accumulation of human capital and social capital of new-type professional farmers not only gives them more opportunities to choose jobs, change careers and start their own businesses in cities but also enables them to get capital accumulation and return home to start their own businesses through rural-urban mobility. The cultivation of new-type professional farmers can bring professional managers' family spirit to the rural economic and social development, integrate social resources to absorb more local employment, promote the adjustment of agricultural industrial structure and economic and social development, and promote the rural revitalization and sustainable development. 


\section{CONCLUSION}

The farmer training project involves many departments, such as the agricultural federation, women's federation, people's society, the Communist Youth League, Township and so on, the total amount of funds invested by the government in the new-type of professional farmers every year is very large, but the input of human, material and financial resources is insufficient to implement into each specific training institution. And some training institutions are driven by economic interests or the requirements of higher authorities to train, so the quality of training can be imagined. In view of this situation, different farmers' training organizations can carry out the following division of labor: rural committee-led, relying on agricultural colleges and universities and agricultural technology schools to carry out skills training for agricultural technology; village committee-led, joint human and social sectors, in collaboration with government departments on the transfer of employment of farmers. Skills training; led by the Bureau of Education, relying on various vocational schools to train basic cultural knowledge, as well as education and training of rural reserve labor force. Through this mode of division of labor, we can distinguish the responsibilities of various departments, mobilize the enthusiasm of all parties, and effectively avoid the shortcomings of repetitive training. Finally, we can form a work promotion mechanism of government overall planning, clear division of labor and mutual cooperation among various departments.

According to the actual needs of national economic development and industrial transformation and upgrading, the standard of vocational skills in the industry is formulated and a unified framework of national skills qualification is constructed. According to the requirement of post competence in industry, we should make a good plan for the curriculum design of vocational training for new-type vocational farmers, innovate the contents and methods of Vocational training, open up the connection channel between vocational training and the career development of new-type vocational migrant workers, improve their ability to start an undertaking and link up with the industrial transformation and upgrading, and shorten the number of actual jobs.

\section{REFERENCES}

[1] Zhong Yang, Liu Keqin. Investigation and Suggestions on the Cultivation of New Professional Farmers [J]. Adult Education, 2018 (10) pp.53-58.(In Chinese)

[2] Yang Ling. Reflections on the cultivation of new-type professional farmers [J]. Cooperative economy and science and technology, 2018 (19): pp. 102-103.(In Chinese)

[3] Wang Qiongjuan. Research on the Restrictive Factors of Training New Professional Farmers and the Countermeasures [J5]. Contemporary Vocational Education:pp.1-5.(In Chinese)

[4] Jinyu. Establishing and improving the basic education system and promoting the cultivation of new professional farmers [J]. Jilin Agriculture, 2018 (19): pp.92.(In Chinese)

[5] Li Yaming, Lu Shumin and Li Weidong. A Brief Talk on the Cultivation of New Professional Farmers in China [J]. Agricultural Science and Technology Communication, 2018 (09):pp.9-12.(In Chinese)

[6] Purong Rong. Talking about how to vigorously develop the cultivation of modern new professional farmers [J]. Agricultural science and technology and information, 2018 (17): pp. 63-64.(In Chinese) 